Cultures \& Conflits

01 | hiver 1990

La prolongation des conflits

\title{
La guerre comme rite géographique : l'exemple de la corne de l'Afrique
}

\section{Alain Gascon}

\section{(2) OpenEdition \\ 1 Journals}

\section{Édition électronique}

URL : http://journals.openedition.org/conflits/78

DOI : $10.4000 /$ conflits.78

ISSN : $1777-5345$

Éditeur :

CCLS - Centre d'études sur les conflits lilberté et sécurité, L'Harmattan

\section{Édition imprimée}

Date de publication : 21 janvier 1990

ISSN : 1157-996X

\section{Référence électronique}

Alain Gascon, «La guerre comme rite géographique : l'exemple de la corne de l'Afrique », Cultures \& Conflits [En ligne], 01 | hiver 1990, mis en ligne le 30 décembre 2002, consulté le 30 mars 2021. URL : http://journals.openedition.org/conflits/78 ; DOI : https://doi.org/10.4000/conflits.78

Ce document a été généré automatiquement le 30 mars 2021.

Creative Commons License 


\title{
La guerre comme rite géographique : l'exemple de la corne de l'Afrique
}

\author{
Alain Gascon
}

Dès 1958, le conflit érythréen commençait par des troubles chez les Beni Amer du bas plateau occidental. En 1960, la Somalie à peine indépendante revendiquait l'Ogaden éthiopien peuplé de Somali, déclenchait deux guerres en 1964 et 1977, et soutenait une longue guérilla pour reprendre la terre irrédente. En avril 1988, invités par le président de Djibouti, Hassan Gouled, Ie président de la Somalie, Siyaad Barre, et le président de l'Ethiopie, Mängestu Haylä Maryam, faisaient la paix à la surprise générale. Or, à peine éteint à la frontière éthiopio-somalienne, l'incendie repartait dès mai 1988 en Érythrée, au Tegré et à l'intérieur de la Somalie. Les rebelles du Nord, du Mouvement national somalien (MNS), prenaient l'offensive et la répression de Mogadiscio atteignait une violence telle que les troupes régulières se mutinaient, démembrant l'État somalien ${ }^{1}$.

La guerre en Érythrée rebondissait après les graves revers subis par l'armée éthiopienne qui avait abandonné le Tegré au printemps. Le 16 mai 1989, Mängestu écrasait un putsch déjoué par ses services secrets, formés par les "spécialistes" de RDA. Peu après, tous les experts d'Allemagne de l'Est et d'Europe de l'Est partaient d'Éthiopie, précédant les Soviétiques gui ont néanmoins exécuté tous leurs contrats de livraison d'armes. Actuellement, en 1990, l'armée ravitaillée par avion ne tient plus que les villes d'Asmarä et de Karän. De tous les côtés, les pouvoirs vacillent, mais tiennent bon, quitte à abandonner les valeurs officiellement reconnues comme légitimes.

Abandonné par ses bailleurs de fonds occidentaux, Siyaad cherche à nouer des contacts avec des États arabes (Libye et Irak) et annonce le pluripartisme. Le 5 mars 1990, Mängestu abandonne le socialisme et le monopole du parti, et donne la terre aux paysans. Il rétablit des relations diplomatiques et militaires avec Israël, et les rebelles du Nord en appellent à la solidarité "arabe" contre Addis Abäba. Bref, les régimes passent, les alliances se nouent et se défont, pourtant les conflits demeurent et compromettent la survie des régimes actuels comme ils avaient précipité la chute des précédents.

La corne de l'Afrique - Un noeud géostratégique "majeur" 
La corne de l'Afrique surveille les routes maritimes de l'Asie et du golfe Persique, elle garde le détroit de Bab-el-Mandeb et le débouché du canal de Suez. Les bases de Djibouti, des îles Dahlak (concédées aux Soviétiques par l'Éthiopie) et de Berbera (concédé aux Etats-Unis par la Somalie) sont les points d'appui indispensables à toute opération vers les champs pétrolifères du Moyen-Orient.

L'Égypte et l'Éthiopie, qui ont chacune 50 millions d'habitants dans une région de peuplement faible et diffus, entretiennent des armées nombreuses et bien équipées, et aspirent à jouer un rôle régional. L'Arabie Saoudite, préoccupée par le sort des musulmans de la rive africaine de la mer Rouge, leur construit de nombreuses mosquées. Elle surveille les relations privilégiées établies entre les deux régimes "marxistes" d'Éthiopie et du Yémen du Sud. Par le port d'Eilat, Israël, encerclé d'États arabes hostiles, maintient une ouverture sur la mer Rouge qui ne doit, à aucun prix, devenir un lac arabe; l'Éthiopie est donc son seul point d'appui où l'État hébreux négocie en outre le retour des Fälasha.

Vue souvent comme l'arrière-cour du Golfe et le prolongement de la rivalité israéloarabe, la corne de l'Afrique est depuis plus d'un siècle, et depuis plus longtemps encore, un centre endogène de "hautes pressions" géopolitiques ravivées par les tensions internationales, comme le montrent les rebondissements des conflits depuis $1988^{2}$. Mais cela suffit-il à en expliquer la nature, l'intensité et la durée ? On peut en douter.

I - Des conflits millénaires et séculaires

Pour expliquer ces conflits, il faut sans doute rappeler que les deux grandes civilisations qui se partagent la corne de l'Afrique portent en elles une conception de l'État dans ses rapports à l'espace qui prend racine dans une vision de l'histoire proche du temps long braudélien ${ }^{3}$. Le poids de l'histoire et des représentations prend alors tout son sens.

Éthiopie : les querelles de légitimité des enfants de la reine de Saba.

Les Éthiopiens s'enorgueillissent d'appartenir à un État qui est indépendant depuis 3000 ans. Ils rappellent qu'ils utilisent le syllabaire en usage dans l'antique royaume d'Aksum, fondé vers le IXème siècle avant notre ère. Ils soulignent leur ascendance israélite par les compagnons du fils de Salomon et de la reine de Saba qui auraient suivi ce dernier à son retour en Éthiopie. Convertis au christianisme au IVème siècle, ils s'affirment donc comme le Peuple élu de l'Ancienne et de la Nouvelle Alliance. Les hautes terres du Nord sont la nouvelle Terre sainte du nouvel Israël. L'enracinement de ce mythe fondateur écrit au XIIIème siècle et peint sur les murs de toutes les églises est si profond que la République socialiste d'Éthiopie a troqué dès sa proclamation en 1987 ses anciennes armoiries imitées de la RDA, un araire, pour les obélisques d'Aksum.

Un héritage aussi vénérable, sanctionné par l'autorité de la tradition, est disputé entre les descendants directs des Aksumites, les Tegréens et les enfants plus lointains, les Amhara. Le particularisme tegréen fut très vivace durant la période gondarienne (XVIIème-XIXème siècle) et se cristallisa dans des lignages "salomoniens" pouvant revendiquer le trône. II s'appuie sur le tegrenna, langue écrite avec le même syllabaire que l'amharique et plus nroche du guèze, la langue d'Aksum. Pour les Tegréens, le déclin commença avec Menilek II qui déposséda les héritiers de Yohannes IV en 1889. Ils lui reprochèrent ses relations avec les Italiens avant son accession au trône et ses alliances avec les chefs oromo. Ils lui firent grief d'avoir cédé aux Italiens, vaincus à 
Adwa, des plateaux du Maräb-Mellash (Outre-Maräb) peuplés de Tegréens, coeur de la colonie d'Érythrée. Ils lui prêtèrent le dessein d'avoir affaibli volontairement le Tegré.

Le règne de Haylä-Sellasé fortifia encore leur ressentiment : il se fit couronner à Addis Abäba et non à Aksum, et tint à l'écart les descendants de Yohannes. Pendant l'occupation, ceux-ci cherchèrent à s'émanciper et la noblesse tegréenne pactisa avec les italiens qui regroupèrent Tegré et Érythrée dans une Grande Erythrée. Les Askaris, supplétifs érythréens qui formaient. Le gros des troupes d'invasion, entrèrent dans les "banda" et pourchassèrent les résistants. A la Libération, les nobles durent se soumettre à nouveau et le gouverneur, ras Abäbä Aragay, d'origine oromo, institua un régime de fer. La province se souleva et la révolte Wayyané fut matée avec le concours de l'aviation anglaise. L'empereur ne visita jamais le Tegré et l'obligea à adopter l'amharique comme langue officielle. Seule concession, il nomma ras, Mangesha Seyum, descendant de Yohannes, devenu son gendre. Erreur, car celui-ci ayant échappé aux exécutions de novembrc $1974^{4}$, fonda au Soudan, le premier Front de libération du Tegré (FLT).

La noblesse foncière du Nord prit la tête du soulèvement populaire provoqué par l'abolition du système traditionnel de tenure dénoncée par l'Église comme un sacrilège (cf. 3). Seulement, des intellectuels marxistes rejoignirent les maquis et évincèrent les aristocrates. Le FLT devint le Front populaire de libération du Tegré (FPLT), Mais l'idéologie marxiste, les sympathies "albanaises" "staliniennes", du front ne l'empêchent pas de s'enraciner dans l'histoire et de manifester une volonté claire de reprendre le

pouvoir usurpé depuis un siècle par les Chaons d'Addis Abäba ${ }^{5}$. Bref, les querelles de légitimité font toujours sens dans les conflits actuels.

Contrôle de la terre et identité culturelle: Grande Ethiopie de Menilek

Menilek II régna de 1889 à 1913 et agrandit plus de deux fois ses territoires, les AmharaTegréens devenant ainsi minoritaires dans la Grande Éthiopie. Cette conquête se fit avec le concours actif des Oromo qui formaient la majorité des soldats d'Adwa. Leurs chefs occupèrent des postes importants à la cour dès le XVIIIème siècle. La mère de Haylä Sellasé était elle-même oromo et sa femme descendait d'un émir du Wallo converti au christianisme. Les populations vaincues devinrent, jusqu'à la réforme agraire de 1975, tenanciers précaires à part de fruit ${ }^{6}$ des "maitres de la terre" venus du Nord ou indigènes amharisés et christianisés. L'octroi d'une concession foncière au Sud fut vécu comrne une sorte de brevet d'éthiopiarité qui faisait entrer l'heuxeux bénéficiaire oromo dans le cercle étroit du peuple élu. Le centre de gravité des soutiens du régime se déplaçait vers les plus récents "éthiopianisés". Avec la chute d'Haylä Sellasé, le système de tenure en vigueur dans le Sud, stigmatisé comme féodal mais qui rappelait bien plus le second servage imposé en Russie au XVIIIème siècle ${ }^{7}$, fut aboli. Cet acte, salué avec enthousiasme au Sud, entraîna la rébellion des gens du Nord, bénéficiaires d'un mode de tenure lignager et communal égalitaire et stable. Il marqua la fin de l'ancien régime ; les cadres privés de leur base économique cédèrent la place à une coalition de paysans et d'étudiants venus "révolutionner" les campagnes pendant la Zämacha ${ }^{8}$. Seulement, les militaires, alarmés par le réveil des nationalités opprimées, réprimèrent brutalement les séparatismes et, tout en maintenant une politique d'alphabétisation en langue locale, promurent l'amharique au rang de "langue de la révolution" suivant le modèle soviétique. Ils justifièrent ainsi les critiques de 
chauvinisme amhara que leur firent les opposants. Le Sud, qui avait applaudi à la révolution, prenait ses distances.

La réforme agraire de 1975 n'était pourtant que la première étape d'une mise au pas des campagnes. Éleveurs et cultivateurs étaient expulsés pour faire place à de grandes exploitations mécanisées qui, nationalisées, devinrent des fermes d'État, vitrines de l'agriculture socialiste scientifique ${ }^{9}$. Les évictions d'alors engendrèrent des troubles qui alimentèrent les guérillas somali et oromo du Bale et d'Ogaden. La Révolution verte, lancée en 1979, annonçait la collectivisation des exploitations et la priorité aux fermes d'État qui reçurent cadres, crédits et machines. Les terres en "surplus" revinrent à l'État qui les redistribua aux fermes et aux réfugiés réinstallés du Nord. Pour prévenir la reprise des troubles, le régime entreprit la villagisation des provinces stratégiques: le Harär, le Rift et le Choa autour d'Addis Abäba. Ce contrôle de la terre au nom du socialisme provoqua dans des sociétés attachées à leurs terroirs et à leurs identités de profondes réactions. Les paysans protestèrent avec leurs pieds et entamèrent un exode vers le Soudan et la Somalie.

Des mouvements à base ethnique du Sud entretinrent des guérillas périphériques. Celles-ci, de moindre importance, furent néanmoins alimentées par des renforts en matériels et en hommes venus des guérillas du Nord. En effet, le FPLT aide le Front de libération des Afar (FLA), des maquis au Gojjam et au Gondär. Quant au Front de libération des Oromo (FLO), il est secouru, via. Le Soudan, par le FPLE. Ainsi, grâce à des aides, les mouvements du Nord créent des diversions sur les arrières de l'armée éthiopienne qui leur seront utiles, notamment lors de leur offensive de l'hiver 1989-1990. Le FLO revendique quant à lui une Oromie indépendante qui rassemblerait des céréaliculteurs chrétiens et partiellement amharisés à l'ouest et musulmans à l'est, des planteurs de café et de faux-bananiers du Sud, des pasteurs islamisés d'Ogaden, revendiqués par la Somalie, et des Walloé amharisés, agro-pasteurs musulmans. Formant $40 \%$ de la population éthiopienne, les Oromo sont les acteurs silencieux de l'histoire de l'Éthiopie depuis trois siècles, tour à tour opprimés et oppresseurs. Les oublier, c'est s'empêcher de percevoir les équilibres de pouvoir interne à la Grande Éthiopie. Leur révolte ou leur soutien sont déterminants pour le régime actuel. Il en va de même pour le sentiment chrétien.

La croix contre le croissant ou le syndrome de Grann

Le cliché de l'Éthiopie chrétienne, forteresse-île au milieu d'un océan musulman, recouvre une réalité qui est perçue par les Éthiopiens comme une épreuve envoyée par Dieu au peuple élu. Les rares invasions qui atteignirent le coeur de l'Éthiopie partirent des basses terres chaudes assimilées à l'enfer. Elles furent menées par des nomades éleveurs musulmans tel l'émir Grann de Harär fléau de Dieu, qui rassembla Somali, Afar et Oromo dans une jihad qui submergea les hautes terres éthiopiennes au XVIème siècle. L'Éthiopie demanda alors l'aide des puissances chrétiennes et les Portugais envoyèrent des soldats. Mais les Européens tentèrent par la suite de ramener l'Église orthodoxe dans l'obédience romaine. Depuis ce temps, l'Éthiopie souffre du syndrome de Grann : elle est obligée de s'isoler pour conserver son identité, mais doit néanmoins maintenir des contacts avec les Européens chrétiens pour lutter contre le danger musulman.

Pendant des siècles, le rôle de filtre, d'État tampon, fut dévolu à des États musulmans périphériques tributaires. Mais cette stratégie avait ses limites puisque Grann était en principe un de ces États qui devait protéger le coeur éthiopien des musulmans. 
Néanmoins, il ne faut pas exagérer l'antagonisme. Les Éthiopiens ont toujours côtoyé les musulmans qui forment, dans toutes les agglomérations, de petites communautés de commerçants et d'artisans parfaitement assimilés. Les marchés urbains sont des enclaves religieuses dominées par les minarets des mosquées, tels Islambét à Gondär ou le Mercato d'Addis Abäba.

Menilek, puis Haylä Sellasé, qui contrôlaient les foyers musulmans des hautes terres, Harär et Jimma, afin de limiter le risque d'une nouvelle jihad, conclurent le même modus vivendi, mais avec les puissances coloniales établies sur les côtes. Quand ils virent, en 1935-1936, les troupes italiennes composées en majorité de contingents indigènes musulmans (Érythréens, Somaliens et Libyens), les régimes éthiopiens furent convaincus de la nécessité de dominer le rivage de la mer Rouge.

En effet, les Italiens s'instituèrent protecteurs de l'islam au Harär et en Érythrée afin d'extirper l'influence de l'orthodoxie. Ils exécutèrent l'abuna, chef de l'Église, des prêtres et des moines et incendièrent maints sanctuaires. L'association Islam, périphérie du nord - agresseurs italiens forgea le sentiment d'être une fois de plus assiégée. Et. Pendant le conflit somalo-éthiopien de 1977-1978, les militaires révolutionnaires, isolés face à la menace d'un nouveau Grann, acceptèrent l'aide qui se proposa, comme le fit le roi du XVIème siècle, mais, cette fois, elle était soviétique ${ }^{10}$. Centre Croix et marxisme se retrouvaient contre l'Islam périphérique.

II Les règlements sociaux des conflits et leurs dérèglements

A cet égard, c'est bien dans la longue durée qu'il faut comprendre les conflits de la Corne. Le marxisme, les renversements d'alliance, les enjeux stratégiques ont certes leur importance, mais ils s'enracinent sur des lignes de clivage bien plus anciennes qui structurent la conflictualité, et les choix des acteurs dans les polarisations antagonistes.

Des guerriers plutôt que des soldats

Chez les Ethiopiens ou chez les Somali, Afar, Oromo, etc., le guerrier et le prêtre (le religieux) représentent les valeurs sociales les plus élevées. Il n'y a pas de frontière entre les deux fonctions : le mad Mullah snmali Maxamed Cabdulle Xasan ${ }^{11}$ mena la guerre contre les Anglais de 1899 à 1920, et Téwodros II régna sur l'Éthiopie de 1855 à 1868, autant comme chef militaire que comme leader religieux.

Les poètes somali, dont le fameux Maxamed Cabdulle Xasan, célèbrent leurs victoires sur des soldats assoiffés et empêtrés dans leur équipement. Avant de partir au combat, le guerrier éthiopien convoqué par les tambours royaux avec sa parentèle, danse et chante son fokkärä devant son chef en lui promettant de lui amener son ennemi couvert de chaînes. Et effectivement, la corne de l'Afrique est le tombeau des armées régulières vaincues par la conjuration des éléments (aridité ou déluges), du relief et de la mobilité des guérilleros complices de ce milieu.

Pourtant, en dépit de ces actes de bravoure et de bravade, en dépit des difficultés du milieu, la Corne fut malgré tout conquise et partagée. Mais cela ne tient pas à ce que les assaillants disposaient d'une avance technique (les armes à feu de Grann, les avions et les chars des Européens en 1920 et 1935), cela tient surtout au fait que les envahisseurs recoururent toujours à des complicités locales. Grann fut guidé par son épouse éthiopienne et chrétienne, la mystérieuse Däl-Wanbära. Les Italiens jouèrent avec Menilek la carte choane contre Yohannes IV du Tegré, puis contre Haylä Sellasé, la carte tégréenne et musulmane (cf. plus haut) et aussi la carte gojjamé avec ras Haylu, rival dynastique malheureux. Les Somalis, tous musulmans sunnites du même rite, 
locuteurs du même somali et partageant le même idéal de vie nomade, n'en offrent pas moins prise aux dissensions internes entre les clans et les confréries. C'est là la principale faiblesse de ce monde de guerriers où les divisions locales empêchent de grandes actions d'envergure contre un ennemi commun. Elles empêchent les armées régulières de contrôler le terrain mais ne peuvent les vaincre ${ }^{12}$.

La guerre comme "rite géographique" régulateur ${ }^{13}$

A ce titre, la guerre apparaît comme un rite géographique régulateur. En effet, dans la Corne, les affrontements, réguliers dans l'espace et dans le temps, absorbent les tensions politiques et sociales. En Éthiopie, les opposants, les shefta, se réfugient dans les forêts chaudes des vallées profondes et des piémonts ou sur les hauteurs glacées. Ils mènent la vie aventureuse des shefta, bandits bien aimés, et rassemblent leurs partisans pour enfin prendre le pouvoir comme le fit Kassa, ou comme Téwodros II, qui obligea Menilek II lui-même à prendre le maquis chez les Oromo. Les Oromo, les Afar (Danakil) et les Somali du sud et de l'est de la Corne ont conservé une structure clanique, très forte pour les deux derniers, dans un système de parenté patrilinéaire (ambilinéaire chez les Amhara-Tegréens). Le système politico-religieux gäda, caractérisé par la succession au pouvoir tous les cinq ans, de huit classes d'âge en deux cycles, est tombé en désuétude chez les Oromo agriculteurs, mais il survit encore chez les éleveurs qui s'affrontent en combats rituels d'initiation où la capture des troupeaux adverses est le gage du courage du jeune initié. Ces razzias de bovins engendrent d'ailleurs des troubles mal tolérés par l'armée éthiopienne ou kényane et pourraient dégénérer ou servir de prétexte à des incidents frontaliers.

Les Afar, quant à eux, sont divisés en groupes tribaux alliés par le système du mariage des cousins croisés. Avant de convoler, le guerrier se doit d'affronter d'autres hommes en des combats à propos des troupeaux et des terrains de parcours. Les Somali appartiennent à une hiérarchie de confédérations, de clans et de lignages jusqu'au reer, segment minimal de parenté. Localement, ils se réunissent, même s'ils appartiennent à des clans différents, en shir, assemblée où ils concluent le xeer, pacte par lequel ils s'engagent à payer le prix du sang pour défendre les pâturages pendant la courte sécheresse entre les deux saisons humides. A la suite de ces guerres, une nouvelle répartition des terrains de parcours s'instaure. Jamais ces affrontements aussi sévères qu'ils aient été, ne mettaient en péril l'existence des groupes, ils manifestaient la nouvelle répartition des forces et des alliances. Les nomades ont préservé ces structures jusqu'à ce qu'éclate la crise climatique et politique récente qui les a précipités, non vers les périmètres irrigués où les attendaient les aménageurs, mais dans les camps et les bidonvilles.

Cette "socialisation par la guerre" la rend normale, habituelle. L'honneur passe avant la paix. Le répertoire de la vengeance, de la violence ouverte est socialement légitime. Il diffracte le territoire et perpétue les systèmes d'opposition, rendant impossible toute unification, même par la force. Cela est vrai pour l'Éthiopie, c'est aussi le cas pour la Somalie dont on vente pourtant l'homogénéité.

La dérive de la Somalie

Les Somali, les Afar et les Oromo se retrouvent de part et d'autre de frontières issues d'accords entre les puissances coloniales et l'Éthiopie.

La Somalie formée des anciennes possessions anglaise et italienne, seul État homogène de la Corne, a dénoncé, en bloc, l'héritage colonial. La récupération des terres irrédentes "cimenta" les Somali autour de Siyaad Barre qui prit le pouvoir en 1969. Il 
décida d'adopter le socialisme scientifique, d'extirper le tribalisme, fit exécuter des sheykh hostiles à l'écriture du somali en caractères latins et soutint les guérilleros du Front de libération de Somalie occidentale (FLSO). Ébranlé par la sécheresse, le régime adhéra à la Ligue arabe en 1974 pour se concilier l'Arabie Saoudite, vers où se dirigent $90 \%$ des exportations somaliennes (produits de l'élevage).

Siyaad Barre attaqua l'Ogaden en 1977 et, lâché par l'URSS, ne reçut qu'une aide occidentale limitée. Son armée dut se retirer derrière la frontière et lui demanda des comptes : il rompit avec les officiers issus de Majerteen qui passèrent en Éthiopie. Il s'appuya alors sur la confédération Daarood et des segments des clans du Nord, Dir et Isaaq : le système MOD où jouaient les liens familiaux ${ }^{14}$. Ses proches investirent les postes les plus élevés et les plus lucratifs et le clan, auparavant chargé de gérer les ressources d'un territoire, devint le canal de redistribution de l'aide internationale captée au plus haut niveau. Bref, l'illusion nationale et l'homogénéité du peuple somalien ne dura qu'un temps.

La base clanique du régime se rétrécit encore avec la rébellion des Isaaq du MNS qui s'appuyèrent sur l'Éthiopie pour conserver l'accès aux pâturages du Hawd que leur interdisaient les Ogaadeen du $\mathrm{FLSO}^{15}$. Les mécanismes de violence et de vengeance, non régulés par le haut, produisirent leurs pleins effets et, à l'heure actuelle, la base du pouvoir est totalement atomisée. Elle ne tient plus qu'à des liens personnels. La diffraction du politique, sa résorption sur des bases territoriales proches des lignes segmentaires et claniques (mais non réductibles à elles), a atteint une intensité inégalée ailleurs. Le seul espoir de Siyaad Barre restant que les multiples fronts sont aussi atomisés que sa propre base sociale.

Dans ce contexte général de la Corne, d'une atomisation par la violence, il est bon de revenir plus en détail sur le principal conflit que connaît la situation érythréenne.

III - L'Érythrée : la Palestine de la corne de l'Afrique

Une colonie et une fédération avortées

La colonie Érythrée des Italiens est l'avorton d'un empire colonial : l'extrémité des hautes terres peuplées de Tegréens, chrétiens agriculteurs, un littoral parcouru par des Afar nomades et musulmans, des montagnes au nord et un bas plateau à l'ouest, mosaïque ethnique (Beni Amer, Tigré, Bariya et Kunama...) et mosaïque religieuse (chrétiens et musulmans) où des éleveurs dominaient des cultivateurs.

Colonie abandonnée, elle servit de tremplin à la conquête de l'Éthiopie pour laquelle la moitié de la population active fut mobilisée (cf. plus haut). Les missions catholiques et protestantes formèrent une élite qui s'opposa à l'Italie (Abba Jérôme, Ato Lorenzo Teezaz) ou collabora (Afä Warq äbrä Iyyäsus) tour à tour ; maintenant, on note le même va-et-vient entre l'administration éthiopienne et la rébellion (Tädla Bayru) ${ }^{16}$.

Sous administration britannique de 1942 à 1952, l'Érythrée, prise en charge par l'ONU comme les colonies italiennes, fut fédérée à l'Éthiopie après consultation des populations et de leurs dirigeants ${ }^{17}$. Un exécutif désigné par un parlement élu au suffrage universel ne laissait à l'Éthiopie que la Défense et la Diplomatie et possédait son propre drapeau. Le tegrenna et l'arabe devinrent langues officielles. La fédération d'un État "démocratique" à une autocratie apparut vite comme une verrue sur la face de l'Éthiopie et aussi un mauvais exemple. Par des prébendes et par des pressions, le gouvernement vida la fédération de sa substance (abandon du drapeau en 1958) et le Parlement vota l'annexion en 1962. L'Érythrée devint le quatorzième gouvernement 
général de l'empire, et l'amharique, la seule langue officielle. Les premiers troubles avaient éclaté quatre ans plus tôt.

Un conflit qui résume tous les autres

Les premiers rebelles érythréens furent des nomades musulmans des basses terres qui reçurent l'aide de l'Égypte et du Soudan au nom du panarabisme et de l'islam. Les cadres venaient de la Ligue musulmane d'Érythrée fondée en 1946, dont une partie des leaders avaient fait cause commune avec le Parti unioniste, appuyé par l'Église orthodoxe et financé par l'Éthiopie. Ces chefs faisaient partie de confréries musulmanes rivales comme au Soudan et en Somalie.

Le gouvernement impérial ne répugnait pas à engager des shefta afin de créer l'insécurité en Érythrée sous administration anglaise pour les combattre ensuite. Il bénéficia de multiples complicités pour rogner l'acte fédéral : l'empereur refusa par trois fois de l'abroger avant d'accepter. Il exploita le ressentiment des "serfs" chrétiens contre les pasteurs musulmans. Le earactère arabe du Front de libération d'Érythrée (FLE) écarta de lui les intellectuels tegréens et chrétiens d'Asmära qui le rejoignirent mais pour mieux s'en détacher et fonder à Damas, au contact des Palestiniens de G. Habbache, le FPLE.

Les deux fronts menèrent une longue guerre sur le terrain et dans les camps du Soudan qui abritent près de un million de réfugiés ${ }^{18}$ Le FPLE a chassé le FLE de ses positions, sauf dans son sanctuaire de l'Ouest. I1 encadre les populations des "territoires libérés" par une organisation politico-militaire inspirée du FI.N et imitée par le FPLT. Un réseau d'ateliers, d'hôpitaux, d'écoles et de routes adossé à Port-Soudan lui assure une autonomie complète même vis-à-vis de ses "hôtes". Une diaspora repliée en Italie et aux Etats-Unis le finance et forme un lobby qui travaille les médias et les politiciens pour la cause érythréenne. Un véritable système de guerre s'est mis en place avec sa propre économie, ses propres structures...

Intransigeant vis-à-vis de ses adversaires, comme le fut Haylä Sellasé ou comme l'est Mängestu, le FPLE montra la même habileté manoeuvrière que les dirigeants éthiopiens dans sa capacité à permuter les alliances.

L'empereur avait offert aux Etats-Unis la base de Qagnnäw en Érythrée pour obtenir leur soutien. Mais, à partir de 1961, il se rapprocha aussi de l'URSS et de la Chine qui soutenaient déjà le FPLE, alors marxiste. II courtisa Nasser et l'Arabie Saoudite et les détacha des fronts érythréens. Le FPLE, pris au dépourvu par la révolution éthiopienne, participa à des rencontres organisées par les Cubains et les États arabes progressistes. Castro proposa une fédération de la corne de l'Afrique, regroupant la Somalie, l'Éthiopie et le Yémen du Sud, qui échoua quand les hostilités éclatèrent en Ogaden. L'armée éthiopienne équipée et entraînée par les Soviétiques reconquit l'Érythrée utile, mais ne délogea pas le FPLE de son sanctuaire adossé au Soudan, sous Nimeiry (1982). Jamais les États frères socialistes ne fournirent au gouvernement de Mängestu les moyens d'éradiquer la résistance érythréenne. Celle-ci conserva toujours la sympathie des États arabes, tant progressistes que conservateurs, qui ne supportaient pas l'alliance Croix et marxisme. L'engagement des Etats-Unis au Soudan permit des contacts officieux par le biais d'ONG. Le FPLE débarrassa son langage des références marxistes et son secrétaire général, Issayas Afäwarqi, parcourut les pays occidentaux où la diaspora érythréenne répercuta le nouveau message. L'inversion idéologique permit aux dirigeants du FLPE de se maintenir au pouvoir dans leur zone et d'éviter toute contestation interne. 
Vainqueur sur le terrain, le FPLE, allié au FPLT, dénonce l'autonomie des régions récemment octroyée par Mängestu et profite de la moins bonne adaptation de son adversaire à la nouvelle donne internationale. En revanche, sur la donne interne, le gouvernement a rallié les Afar, certains clans somali en Ogaden et des notables du FLE, partisans d'un partage chrétien musulman Érythrée. Les développements récents (implication d'Israël, en départ des Soviétiques et offensives des fronts au Nord) montrent que le "feuilleton" érythréen continue, même si la distribution des rôles change.

Une, deux, trois Érythrée dans la corne de l'Afrique?

Protéiformes à travers le temps et l'espace, les conflits furent et sont encore une lutte pour la domination d'un territoire dans un espace fluide dans les basses terres et figé sur les hautes terres. Chez les nomades les limites se jouaient dans des conflits rituels et réguliers dans des structures claniques fortes. Chez les Amhara-Tegréens, les conflits étaient censés se dérouler à la périphérie d'un État sanctuaire théocratique fort d'une légitimation par le temps long. L'espace fluide des Somali est balisé seulement par le réseau des tombeaux de saints de l'Islam.

L'intervention des puissances planétaires ou régionales a perturbé ces rites géographiques en donnant à certains protagonistes des moyens de rendre les résultats des conflits irréversibles. Menilek, menacé, tel l'empereur Meiji, utilisa les armes de ses adversaires européens pour survivre et défaire ses partenaires africains, mais toléra les Italiens en Érythrée pour affaiblir le Tegré, comme on tolérait naguère des shefta contre ses concurrents. Avec la guerre italo-éthiopienne, la décolonisation et la tension Est-Ouest, les shefta s'effacèrent devant les arbännoch, les patriotes, et shefta prit même un sens péjoratif.

L'Érythrée est l'exemple de la mutation d'un conflit qui se régénère au gré des interventions extérieures et se radicalise sur le terrain: destructions de vivres et d'équipements, déplacements de population exécutés par tous les belligérants. Le conflit du Tegré prend lui aussi ce tour "sendériste" avec les mêmes exactions, y compris à l'égard des ONG. Est-ce là l'avenir de ces conflits ? Jusqu'où ira l'atomisation de la violence ? La reproduction sur longue durée des conflits n'empêche pas, semble-til, leurs transformations, leurs métamorphoses ${ }^{19}$. C'est dans ce rapport entre les métamorphoses conflictuelles et la perpétuation des lignes de clivage que s'explique à la fois la durée et les variations d'intensité des conflits.

\section{NOTES}

1. Cf. mes articles dans l'Etat du Monde 1988, 1989, 1990 et 1991 et Etudes Polémologiques, $n^{\circ} 51,3 / 1989$. La translittération des noms propres éthiopiens suit le plus possible la prononciation locale, on a respecté l'orthographe et les voyelles redoublées en somali.

Aw en somali : sheykh. Grann en amharique : Gurey en somali (le gaucher). Tegre en amharique : Tigray en tegrenna. 
2. J'emprunte la comparaison "hautes pressions" à Ancel J., Géographie des Frontières, Paris, NRF, 1938.

3. Gascon A., Etat contemporain, Etat ancien : l'Ethiopie, in E. Terray, l'Etat contemporain en Afrique, Paris, L'Harmattan, 1987, pp. 141-156, et Gascon et Hirsch B., Naissance d'une Frontière : un siècle de conflit somalo-éthiopien in La géopolitique des militaires, des diplomates et des professeurs, colloque organisé par P. Claval, Paris, 1990, à paraître.

4. Les anciens ministres, les chefs militaires et des princes détenus aux Vieux Palais furent abattus le 23 novembre 1974.

5. Cf. l'interview à la BBC (automne 1989). Son alliance actuelle avec le Front populaire de libération d'Erythrée (FPLE) a contribué à passer sous silence la question de Tegréens qui font partie de l'Erythrée qui réclame l'indépendance.

6. Le propriétaire de la concession recevait la moitié ou le quart de la récolte (fruit de la terre).

7. Fascinés par la théorie des cinq stades, des auteurs éthiopiens ne connaissent pas le mode de production asiatique.

8. La campagne d' alphabétisation et de développement par la coopération lancée en 1975-1976 par le gouvernement militaire provisoire.

9. Gascon A., Le sabre et le tracteur. Les fermes d'État dans l'agriculture socialiste d'Éthiopie, Bulletin des Etudes africaines de l'Inalco , vol. VII, nº 13-14, 1987, p. 237 à $248,1989$.

10. Cette référence à Grann et aux Portugais était explicite dans l'argumentation des Éthiopiens quand fut conclue l'alliance avec l'URSS.

11. Orthographié plus fréquemment Mohamed Abdul Hasan.

12. La victoire de Menilek à Adwa est, à cet égard, un événement exceptionnel.

13. Cette formule est empruntée à Bonnemaison J., Communication au colloque Ethnogéographies, Bordeaux (CEGET), P. Claval et P Siregaravelou, 1990.

14. Lewis I.M., A Modern History of Somalia : Nation and State in the Horn of Africa, Harlom, Longman, 1982. Le système MOD repose sur de clan Mareehan de la mère de Siyaad, le clan Ogaadeen de sa deuxième épouse et le clan Dhul banhante de son gendre. Laitin DD. Et Sarnantar S.S., Somalia. Nation in Search of State, Boulder (Colorado), Westview Press, London, Gomer 1987.

15. Markahis J. National and Class Conflict in the Horn of Africa, Cambridge, African Studies Series 55, Cambridge Universily Press, 1987. Aquarone M.C., Les frontières du refus : six séparatismes africains, Paris, Mémoires et Documenls du CNRS, Éd. Du CNRS, 1987, $142 \mathrm{p}$.

16. Marchal R., Production sociale et recomposition politique dans l'exil : le cas érythréen, Cahier d'études africaines, XXVII 3-4, 1987, p. 393-400. Rouaud A., Afäwarq Gäbrä Iyyäsus Thèse, Inalco, 1988. Spencer J. H., Ethiopia at Bav : A personnal of the Haile Sellasie Years, Algonac, Reference Publications Inc., 1987. Tesfatsion Medhanie, Eritrea : Dynamics of a National Question, Amsterdam, B. R. Grüner, 1986.

17. Rossij G., L'africa italiana verso l'independenza, Roma, Giuffrè editore, 1980.

18. Shoa Asfeha, De l'Erythrée au Soudan. Contribution géographique à l'étude d'une politique d'assistance aux réfugiés, thèse de doctorat de l'EHESS, 1988.

19. Bigo Didier et Hermant Daniel, La métamorphose des conflits, Etudes Polémologiques, $\mathrm{n}^{\circ}$ 50, 2/1989, pp. 5-20. 


\section{RÉSUMÉS}

La corne de l'Afrique est le théâtre de conflits qui ont commencé avant même la fin de la période coloniale. Ils se poursuivent alors que l'URSS, ses ex-alliés et les Occidentaux se dégagent de toute intervention directe au côté d'un des belligérants. Depuis 1988 la tension intérieure a bien diminué : la Somalie et l'Ethiopie ont fait la paix, Mangestu a rendu la terre aux paysans, renoncé au parti unique et renoué avec Israël et Siyaad appelé au partage du pouvoir. Pourtant la guerre ravage la Somalie, seul Etat dit homogène de la Corne, guetté par le démembrement. En Ethiopie, le régime fait face aux rebelles tegréens qui, alliés aux Erythréens, menacent la capitale. Il parvient, malgré tout, à se maintenir, est-ce seulement à coup de concessions ? N'aurait-il pas une certaine légitimité ? 3000 ans d' "Histoire Sainte " pèsent plus que 15 ans de Révolution futelle marxiste!

The Horn of Africa has been the scene of conflicts already started before the end of the colonial period. They are perpetuating while the USSR, its former allies and the Western Powers are shunning any direct involvement in any of the belligerent sides. Since 1988, internal tension has abated : Somalia and Ethiopia have come to a peace agreement, Mangestu has restored the Ethiopian peasantry to its rights over the land, given up the one party system and revived relations with Israel while Siyaad has made a bid for the share in the exercise of power. Nevertheless, Somalia, thought to be the only homogeneous state in the Horn, is devastated by war and on the eve of partition. In Ethiopia, the present government has to face the Tegrean rebels who, through their alliance with the Eritreans, are threatening the capital city. It has so far managed to survive, although at the expense of countless concessions. Is it endowed with a certain amount of legitimacy? The weight of 3, 000 years of Biblical history is heavier than 15 years of a Revolution, be it a Marxist one !

\section{INDEX}

Index géographique : Corne de l'Afrique

Mots-clés : anthropologie, conflits, guerre, territoire(s) et territorialité, géographie

\section{AUTEUR}

\section{ALAIN GASCON}

Professeur d'histoire et de géographie, chercheur au laboratoire de langues et sociologies africaines, URA 94 (CNRS-EHESS) 